\title{
Syndrome d'encéphalopathie postérieure réversible ou Posterior Reversible Encephalopathy Syndrome (PRES) secondaire au bevacizumab
}

\author{
Posterior Reversible Encephalopathy Syndrome (PRES) due to bevacizumab
}

\author{
O. Peyrony $\cdot$ C. de Margerie-Mellon · E. Mariotte
}

Reçu le 11 août 2017 ; accepté le 29 septembre 2017

(C) SFMU et Lavoisier SAS 2017

\section{Introduction}

Le syndrome d'encéphalopathie postérieure réversible ou Posterior Reversible Encephalopathy Syndrome (PRES) a été décrit pour la première fois en 1996 par Hinchey et al. [1] qui ont rapporté un syndrome combinant céphalées, confusion, convulsions et perte de la vision associées à des images de leuco-encéphalopathie sur le scanner ou sur l'imagerie par résonance magnétique (IRM). L'hypertension en est une cause fréquente, mais d'autres étiologies telles que l'éclampsie, les maladies inflammatoires chroniques ou certains médicaments cytotoxiques, sont possibles. Le bevacizumab est un anticorps monoclonal inhibant le Vascular Endothelial Growth Factor (VEGF) indiqué dans le traitement de certains cancers et notamment celui du sein métastatique [2]. Si l'hypertension artérielle est un effet secondaire fréquent de ce traitement, le PRES est, lui, beaucoup plus rare. Nous rapportons ici un cas de PRES secondaire à la prise de bevacizumab.

\section{Observation}

Une patiente de 54 ans, suivie pour un cancer du sein métastatique à l'os et traitée par 12 cycles de bevacizumab, consulte aux urgences vers 22 heures pour confusion, cécité

\footnotetext{
O. Peyrony $(\bowtie)$

Service des urgences, hôpital Saint-Louis,

Assistance Publique-Hôpitaux de Paris (APHP),

1 avenue Claude Vellefaux, 75010 Paris, France

e-mail : olivier.peyrony@aphp.fr

C. de Margerie-Mellon

Service de radiologie, hôpital Saint-Louis, AP-HP,

1 avenue Claude Vellefaux, 75010 Paris, France

E. Mariotte

Service de réanimation médicale, hôpital Saint-Louis, AP-HP,

1 avenue Claude Vellefaux, 75010 Paris, France
}

et céphalée ayant débuté brutalement le jour-même. Elle ne présente pas d'autre comorbidité ou d'intoxication alcoolotabagique et ne prend aucun autre traitement. Les paramètres vitaux initiaux sont sans particularité hormis une hypertension modérée à $166 / 87 \mathrm{mmHg}$, ainsi qu'une tachycardie à $117 \mathrm{bpm}$. La température est à $37,4^{\circ} \mathrm{C}$ et la glycémie capillaire est normale. Hormis la confusion et la cécité, l'examen neurologique ne montre pas d'anomalie (il n'y a pas de signe de localisation, les pupilles sont symétriques et réactives, il n'y a pas de syndrome pyramidal et la nuque est souple). La patiente ne présente ni ictère, ni asterixis, ni signe d'insuffisance hépatocellulaire en faveur d'une encéphalopathie hépatique. Il n'y a pas de trouble ventilatoire et l'auscultation pulmonaire est normale, rendant peu probable une encéphalopathie hypercapnique. Dans l'hypothèse de localisations cérébrales secondaires, un scanner cérébral est réalisé qui ne montre pas d'anomalies hormis des métastases du crâne déjà connues et n'expliquant pas le tableau clinique. La biologie ne montre pas de dysnatrémie, d'hypercalcémie, d'anomalie du bilan hépatique ou de stigmate de microangiopathie thrombotique pouvant expliquer les symptômes. Vers minuit, la patiente présente un épisode de perte de contact associé à des mouvements tonico-cloniques généralisés, résolutifs après $1 \mathrm{mg}$ de clonazépam. Une élévation de la température à $38,4^{\circ} \mathrm{C}$ motive la réalisation d'une ponction lombaire. L'analyse du liquide céphalorachidien, normale, élimine une méningite bactérienne et rend peu probable une méningo-encéphalite métastatique. Dans la matinée, une IRM cérébrale est réalisée. Elle montre des hypersignaux bilatéraux asymétriques dans les régions corticales et souscorticales des lobes temporo-occipitaux sur la séquence Fluid Attenuation Inversion Recovery (FLAIR) évoquant le diagnostic de PRES (Fig. 1A, B).

La patiente est alors transférée en réanimation médicale où un traitement antihypertenseur parentéral est débuté avec une résolution rapide des symptômes. L'étiologie toxique est retenue et le bevacizumab n'est pas repris lors des chimiothérapies suivantes. 


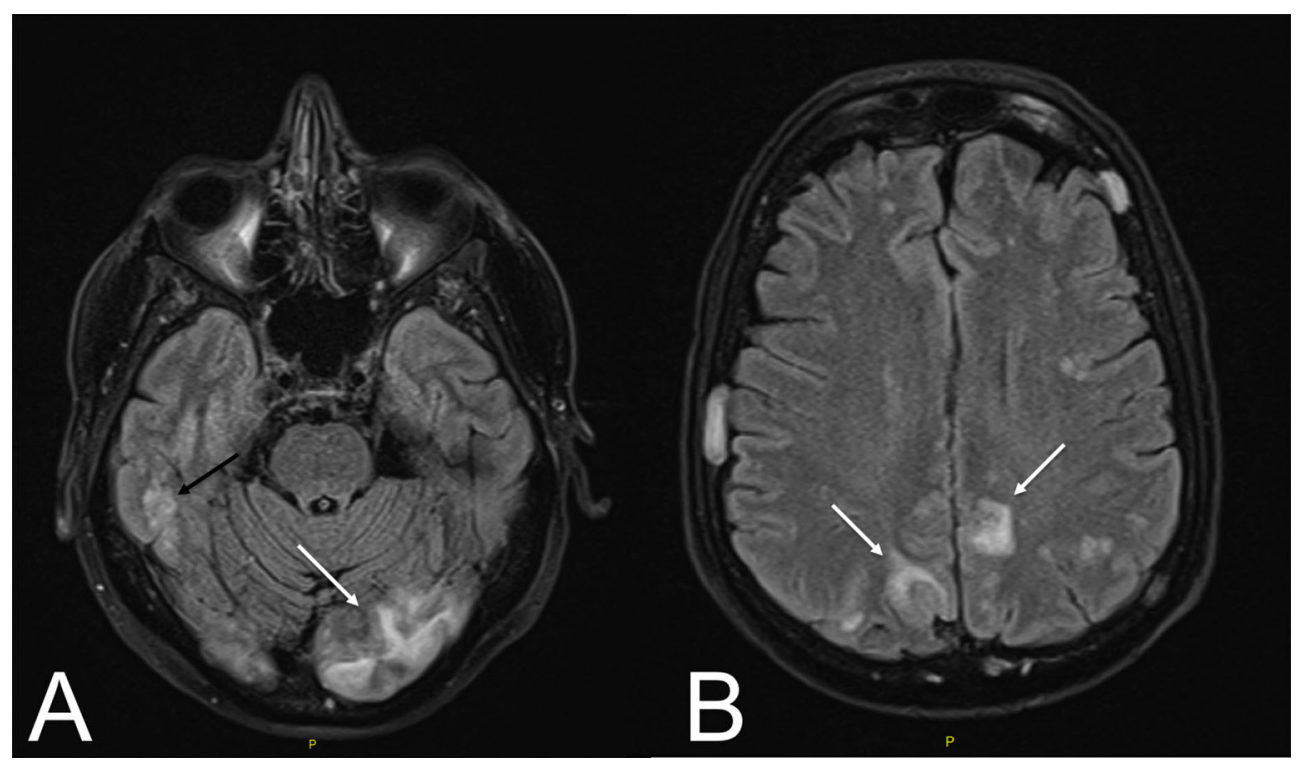

Fig. 1 Imagerie par résonnance magnétique cérébrale (coupe axiale, séquence T2 FLAIR) montrant des hypersignaux bilatéraux asymétriques dans les régions corticales et sous-corticales des lobes temporaux (A : flèche noire) et occipitaux (A, B : flèches blanches) faisant évoquer le diagnostic d'encéphalopathie postérieure réversible ou Posterior Reversible Encephalopathy Syndrome (PRES)

\section{Discussion}

Le PRES est lié à un œdème cérébral vasogénique responsable de symptômes neurologiques aigus tels qu'une encéphalopathie (50-80 \%), une crise convulsive (60-75\%) pouvant se compliquer d'un état de mal épileptique (5-15\%), une céphalée $(50 \%)$, des anomalies de la vision à type de cécité corticale, de vision floue, de défect du champ visuel ou d'hémianopsie latérale homonyme (33\%) ou un déficit neurologique focal (10-15\%) [3]. La fièvre, comme dans notre cas clinique, n'est habituellement pas décrite et peut éloigner le clinicien en l'orientant vers une étiologie septique.

Bien que des anomalies soient parfois visibles sur le scanner cérébral sans injection (hypodensités postérieures bilatérales) [1,4], l'IRM est l'examen de choix. Elle montre habituellement un œdème des régions sous-corticales (et parfois corticales) intéressant les lobes pariétaux et occipitaux, caractérisé par un hypersignal asymétrique sur la séquence T2FLAIR. Les lobes frontaux, le cervelet et le tronc peuvent également être concernés. Des complications de type ischémique ou hémorragique (intra-parenchymateuse ou sousarachnoïdienne) sont possibles dans $15-30 \%$ et $10-25 \%$ des cas respectivement [3].

Le PRES est généralement réversible en une semaine mais un décès peut survenir ou des séquelles neurologiques peuvent persister chez certains patients en cas d'hémorragie ou d'infarctus cérébral [3]. Les autres facteurs de mauvais pronostic cités dans la littérature sont un retard dans le contrôle de l'étiologie du PRES et une hyperglycémie au premier jour [5].
La physiopathologie du PRES reste incertaine et voit deux théories s'opposer. La première repose sur un phénomène d'hyperperfusion secondaire à une poussée hypertensive. En effet, dans le cas de l'hypertension artérielle, qui est une des étiologies les plus fréquentes de PRES, l'élévation de la pression artérielle (PA) systémique entraîne une hyperperfusion cérébrale par dépassement des mécanismes d'autorégulation de la perfusion cérébrale et conduit à une altération de la barrière hémato-encéphalique avec extravasation de plasma ou de sang $[3,4]$. La région postérieure est plus sensible à ces phénomènes d'hyperperfusion du fait d'une moindre innervation sympathique $[3,4]$. Dans plus de la moitié des cas, les patients ont une PA modérément élevée (PA moyenne inférieure au seuil supérieur d'autorégulation de la perfusion cérébrale de $140-150 \mathrm{mmHg}$ ) et dans 15 à $20 \%$ des cas, la PA peut être normale ou basse [3]. Il semblerait donc que l'élévation rapide de la PA soit plus délétère que sa valeur absolue. Le traitement repose sur un contrôle progressif de la PA pour éviter des phénomènes d'ischémie cérébrale. Une baisse minime de PA entraîne souvent une amélioration clinique rapide du fait de la relation linéaire entre la pression de perfusion cérébrale et la PA liée à la perte d'autorégulation cérébrale. La nicardipine par voie parentérale est le traitement de première intention [4].

La seconde théorie repose, elle, sur un phénomène de vasoconstriction et d'hypoperfusion entraînant une ischémie et un œdème cérébral secondaires à une toxicité systémique [6]. En effet, des processus inflammatoires chroniques ou aigus ainsi que certains traitements cytotoxiques peuvent être responsables de PRES alors que la PA est peu élevée et 
Tableau 1 Principales étiologies d'encéphalopathie postérieure réversible ou Posterior Reversible Encephalopathy Syndrome (PRES)

Hypertension artérielle

Pré-éclampsie / éclampsie

Agents cytotoxiques et immunosuppresseurs

Toxicité médicamenteuse

Maladies auto-immunes

Sepsis

Anomalies métaboliques

Syndrome de lyse tumorale

Insuffisance rénale aiguë ou chronique

Intoxications

Transfusions massives
Cytarabine, anthracyclines, vincristine, anti-angiogéniques, anticorps monoclonaux, cyclophosphamide (endoxan), méthotrexate, tacrolimus (advagraf), ciclosporine A (neoral)

Antirétroviraux, interféron alpha, corticothérapie haute dose, érythropoïétine, granulocytes colony stimulating factor (GCSF), immunoglobulines

Lupus, sclérodermie, granulomatose de Wegener, polyarthrite rhumatoïde, mico-angiopathie thrombotique

Hypomagnésémie, hypercalcémie

Stupéfiants, venins, digitaliques n'atteint pas le seuil de perte de l'autorégulation cérébrale $[3,6]$. Le Tableau 1 résume les principales étiologies du PRES.

Alors qu'une élévation du VEGF, qui stimule l'angiogenèse et augmente la perméabilité vasculaire, peut favoriser l'œdème vasogénique dans l'éclampsie ou la pré-éclampsie, les traitements inhibant le VEGF tels que le bevacizumab, l'aflibercept, le sorafenib et le sunitinib peuvent également être responsables de PRES. Si l'hypertension est un des effets secondaires les plus fréquemment décrits avec ces traitements, le PRES est beaucoup plus rare. Un des mécanismes incriminés, outre l'hypertension, est une diminution du monoxyde d'azote (NO) circulant qui pourrait affecter la perfusion cérébrale [3]. Le premier cas de PRES secondaire au bevacizumab a été décrit en 2006 par Glusker et al. [7]. En 2011, Tlemsani et al. ont décrit 26 cas rapportés dans la littérature entre 2000 et 2011 de PRES secondaires à la prise d'anti-VEGF [8]. Parmi ces cas, un tiers seulement avait un antécédent d'hypertension artérielle et $92 \%$ des patients étaient hypertendus lors du diagnostic de PRES. En 2015, Singer et al. ont rapporté 31 cas de PRES chez des patients cancéreux parmi lesquels 6 (35\%) étaient sous bevacizumab. Dans cette étude, le nombre médian de doses reçues avant le début des symptômes était de 8 ( $\min : 1-\max$ : 19). Sur la période de l'étude, l'incidence des cas de PRES chez les patients traités par bevacizumab était de $0,1 \%$ [9].

Les patients cancéreux sont donc à risque de développer ce syndrome de par les multiples facteurs de risque auxquels ils sont exposés (chimiothérapies cytotoxiques ou anti-VEGF, traitements immunosuppresseurs chez les patients allogreffés, corticothérapie, micro-angiopathies, hypertension et hyper-hydratation, sepsis, syndrome de lyse, insuffisance rénale aiguë, hypercalcémie, injections de GCSF ou d'érythropoïetine, transfusions...) [10]. De plus, chez ces patients, le clinicien doit également évoquer les causes non spécifiques (accident vasculaire cérébral, thrombose veineuse, méningo-encéphalite septique, causes métaboliques...) ainsi que celles liées à la pathologie tumorale (métastases cérébrales, œdème, hémorragie ou comitialité compliquant des localisations secondaires déjà connues, hypercalcémie, méningo-encéphalite métastatique...) ou liées aux traitements du cancer (chimiothérapies, immunothérapies, corticothérapie, morphine...).

En conclusion, ce cas clinique met en avant ce syndrome rare mais potentiellement grave qui devrait être évoqué par les cliniciens et confirmé par la réalisation d'une IRM en urgence, chez un patient confus ou ayant convulsé et présentant des troubles de la vision, quelle que soit sa PA, ce d'autant plus qu'il présente des comorbidités ou des traitements à risque tels que le bevacizumab.

Liens d'intérêts : Les auteurs déclarent ne pas avoir de lien d'intérêt.

\section{Références}

1. Hinchey J, Chaves C, Appignani B, et al (1996) A reversible posterior leukoencephalopathy syndrome. N Engl J Med 334: 494-500

2. Miller K, Wang M, Gralow J, et al (2007) Paclitaxel plus bevacizumab versus paclitaxel alone for metastatic breast cancer. N Engl J Med 357:2666-76

3. Fugate JE, Rabinstein AA (2015) Posterior reversible encephalopathy syndrome: clinical and radiological manifestations, pathophysiology, and outstanding questions. Lancet Neurol 14:914-25

4. Servillo G, Bifulco F, De Robertis E, et al (2007) Posterior reversible encephalopathy syndrome in intensive care medicine. Intensive Care Med 33:230-6 
5. Legriel S, Schraub O, Azoulay E, et al (2012) Determinants of recovery from severe posterior reversible encephalopathy syndrome. PLoS One 7:e44534

6. Bartynski WS (2008) Posterior reversible encephalopathy syndrome, part 2: controversies surrounding pathophysiology of vasogenic edema. Am J Neuroradiol 29:1043-9

7. Glusker P, Recht L, Lane B (2006) Reversible posterior leukoencephalopathy syndrome and bevacizumab. $N$ Engl $\mathrm{J}$ Med $354: 980-2$
8. Tlemsani C, Mir O, Boudou-Rouquette P, et al (2011) Posterior reversible encephalopathy syndrome induced by anti-VEGF agents. Target Oncol 6:253-8

9. Singer S, Grommes C, Reiner AS, et al (2015) Posterior reversible encephalopathy syndrome in patients with cancer. Oncologist 20:806-11

10. Le EM, Loghin ME (2014) Posterior reversible encephalopathy syndrome: A neurologic phenomenon in cancer patients. Curr Oncol Rep 16:383 